\title{
Monte Carlo comparison of rival experimental designs for two-agent combined action studies
}

\author{
WILLIAM R GRECO PhD, DAVID C SUTOR, JOHN C PARSONS, LEONID A KHINKIS PhD, LILY HSIEH. \\ SOWMYA R RAO, YING TUNG, ChrISTOPHER C CURRIE, ROBIN SUSICE
}

\begin{abstract}
WR Greco, DC Sutor, JC Parsons, et al. Monte Carlo comparison of rival experimental designs for two-agent combined action studies. Can J Infect Dis 1994;5(Supp1 A):51A-59A.

OвJEстіvE: The combined action of two or more chemotherapeutic agents and/or biological agents can be quantitatively described with empirical multidimensional concentration-effect response surface models. This intuitive statistical approach provides a framework for suggesting experimental designs for in vitro, in vivo and possibly clinical experiments of agent combinations. Five rival 32-point experimental designs for in vitro continuous response two-agent combined action studies were compared using Monte Carlo simulation.

Design: The designs were: factorial; central composite; one-ray in duplicate; four-ray; and D-optimal. Serting: Datasets were simulated by generating ideal data with the authors' flagship two-agent combined action model, which includes six parameters: the control survival, Econ=100 (where Econ is the full range of response that can be affected by the drug); median effective concentrations, $I_{50,1}=10, I_{50,2}=1$ for drug 1 and drug 2, respectively; slope parameters, $m_{1}=-1, m_{2}=-2$ for drug 1 and drug 2 , respectively; and the interaction parameter, $\alpha=1$ or $\alpha=5$. For each design, for each of four types of error (absolute, relative with $1 \%$ coefficient of variation [cv], relative with $10 \% \mathrm{cv}$, and relative with $10 \% \mathrm{cv}$ plus a noise constant of $1 \%$ of Econ), for each of two values of the true $\alpha(1,5), 500$ Monte Carlo datasets were generated, and then fit via weighted nonlinear regression with the flagship model.

Marn Results: For the $\alpha$ parameter, for relative error-containing datasets, the D-optimal designs had the smallest variances.

Conclusion: The counterintuitive D-optimal designs may be useful for studies in which the experimental units are relatively precious, and frugal designs are essential. In addition, it may be fruitful to add the D-optimal design points to standard experimental designs.
\end{abstract}

Key Words: Combined action model, D-optimal design, Experimental design, Monte Carlo simulation, Synergy

\section{Comparaison de modèles expérimentaux rivaux pour deux études sur l'action combinée de deux agents}

ОвлестіF : L’action combinée de deux agents chimiothérapeutiques ou biologiques (ou de plusieurs) peut être décrite quantitativement à l'aide de modèles concentration-effet empiriques pluridimensionnels. Cette approche statistique intuitive offre une base pour l'élaboration de modèles d'expérimentation in vitro, in vivo et possiblement, pour les essais cliniques sur les thérapeutiques d'association. Cinq études sur la réponse in vitro continue à des thérapeutiques d'association doubles, à l'aide de modèles expérimentaux en 32 points ont été comparées à l'aide d'une simulation. 


\begin{abstract}
Modèle : Les modèles ont été les suivants: factoriel, central composite, à rayon unique en duplicate, à quatre rayons, et D-optimal.

Contexte : Des séries de données ont été simulées en générant des données idéales à partir du modèle à action combinée de l'auteur qui incluait six paramètres: survie des témoins, Econ = 100 (où Econ représente la gamme complète des réponses qui peuvent être influencées par l'agent); concentrations efficaces médianes, $C_{50.1}=10, C_{50.2}=1$ pour les médicaments 1 et 2 respectivement; paramètres des courbes, $m_{1}=-1$, $m_{2}=-2$ pour les médicaments 1 et 2 respectivement, et paramètre d'interaction, $=1$ ou $=5$. Pour chaque modèle, et chacun des quatre types d'erreurs (absolue, relative avec $1 \%$ de coefficient de variation [cv], relative avec $10 \%$ de cV et relative avec $10 \%$ de cv plus une constante d'interférence de $1 \%$ pour Econ), et pour chacune des deux valeurs du $\alpha$ véritable $(1,5), 500$ séries de données simulées ont été générées et assorties au modèle selon un principe de régression non linéaire pondérée.

Principaux Résultat s: Pour le paramètre $\alpha$, pour les séries de données renfermant des erreurs, les modèles D-optimaux présentaient les variances les plus faibles.

Conclusion : Les modèles contre-intuitifs D-optimum peuvent être utiles aux études où les unités expérimentales sont relativement précieuses, et où les modèles simples sont essentiels. De plus, il peut être avantageux d'ajouter des éléments du modèle D-optimal aux modèles expérimentaux standard.
\end{abstract}

$\mathrm{E}$ FFECTIVE CLINICAL THERAPIES FOR CANCER AND INFEC tious diseases often include combinations of agents. Agent combinations may comprise traditional pharmaceuticals, natural biologicals and/or modified biologicals. The quantitative assessment of the nature and intensity of combined action of mixtures of agents in vitro, in vivo, and especially in clinical studies, can be difficult. The most rigorous approaches to this problem involve the derivation of multiple agent concentration-effect combined action response surface models that include interaction parameters, and the fitting of these models to experimental data with modern statistical curve-fitting approaches. The authors have developed such a set of models and curve-fitting techniques (1-5), and have applied this paradigm to several in vitro studies of combinations of anticancer and other agents against cells in culture (6-9).

\section{THREE-DIMENSIONAL COMBINED ACTION MODELS}

Our flagship combined action model is given as Equation 1. Equation 1 was derived with an adaptation of an approach suggested by Berenbaum (10), with the assumption of Equation 2, the Hill model (11), as the appropriate model for each agent alone.

In Equations 1 and $2, E$ is the measured effect (response), $D$ is the concentration (or dosage) of drug, Econ is the full range of response that can be affected by the drug, IC50 is the median effective dosage (or concentration) of agent, and $m$ is a slope parameter (additional subscripts, 1 and 2, refer to agents 1 and 2, respectively). When $m$ has a negative sign, the curve falls with increasing agent concentration; when $m$ is positive, the curve rises with increasing agent concentration. The interaction parameter is $\alpha$. When $\alpha$ is positive, Loewe synergism is indicated, when $\alpha$ is negative, Loewe antagonism is indicated, and when $\alpha$ is 0 , Loewe additivity is indicated. (This terminology is the central element of a recent consensus on combined action nomenclature and concepts [12]. The use of 'Loewe' as an adjective stresses the connection to the 'no interaction' reference model inherent in the classi- cal isobologram approach to interaction assessment pioneered by Loewe [13].) The magnitude of $\alpha$ indicates the intensity of the interaction. Thus, although Equation 1 is not the model for Loewe synergism (or Loewe antagonism), it is a model for Loewe synergism (or Loewe antagonism).

The derivation of Equation 1, the flagship equation for two-drug interactions, is provided in detail in Greco et al (6). Equation 1 allows the slopes of the concentration-effect curves for the two drugs to be unequal. It is this key feature that distinguishes Equation 1 from many other response surface models used by others to describe drug interactions (eg, 14). Since Equation 1 is in unclosed form, a one-dimensional bisection root finder $(\mathrm{eg}, 15)$ is used to calculate $E$ for simulations. Equation 1 was not derived from biological theory, rather it is an empirical equation which often matches the shape of real data (eg, 2,6-8).

\section{STATISTICAL EXPERIMENTAL DESIGN}

The main decisions that must be made regarding experimental design are: first, where to choose the concentrations; second, numbers of replicates; and third, numbers of experiments. These seemingly simple questions have spawned many full careers for statisticians, who have delved deeply into them to reveal their inherent complexity. The adoption of a response surface paradigm for the assessment of combined action of agents facilitates the understanding and use of formal statistical experimental design. First, the experimenter must decide whether he/she is in an exploratory or a confirmatory mode. Screening experiments (exploratory mode) should first include, for each agent individually, agent concentrations which span the anticipated response region. Logarithmic spacing of the concentrations over a thousand-fold to a million-fold range is probably necessary, depending upon the previous knowledge of the researcher about the concentrationeffect behaviour of the compound. After the individual agent concentration-effect curves are well characterized, a combination experiment should be conducted 


$$
1=\frac{D_{1}}{I C_{50,1}\left(\frac{E}{E c o n-E}\right)^{1 / m_{1}}}+\frac{D_{2}}{I C_{50.2}\left(\frac{E}{E c o n-E}\right)^{1 / m_{2}}}+\frac{\alpha D_{1} D_{2}}{I C_{50,1} I C_{50,2}\left(\frac{E}{E c o n-E}\right)\left(1 / 2 m_{1}+1 / 2 m_{2}\right)} \quad \quad E=\frac{E \operatorname{con}\left(\frac{D}{I C_{50}}\right)^{m}}{1+\left(\frac{D}{I C_{50}}\right)^{m}}
$$

Equation 1

Equation 2

TABLE 1

Factorial design (5X5)

\begin{tabular}{cccccccc}
\hline Drug 2 & \multicolumn{7}{c}{ Drug 1 concentration } \\
concentration & 0 & 2 & 5 & 10 & 20 & 50 & 100 \\
\hline 0 & 4 & 1 & 1 & 1 & 1 & 1 & 1 \\
0.2 & 1 & 1 & 1 & 1 & & 1 & \\
0.5 & 1 & 1 & 1 & 1 & & 1 & \\
1 & 1 & 1 & 1 & 1 & & 1 & \\
2 & 1 & & & & & & \\
5 & 1 & 1 & 1 & 1 & & 1 & \\
10 & 1 & & & & & &
\end{tabular}

Table entries are the number of replicates at each design point, ie, each combination of drug 1 concentration and drug 2 concentration

that repeats single agent data points, and that includes a set of combination points. A full factorial (checkerboard) design (Table 1), a single ray (fixed ratio) design (Table 3) or a multiple ray design (Table 4), all with logarithmically spaced concentrations, may be appropriate. If a complex three-dimensional concentrationeffect surface is anticipated, then the entire interesting region of agent 1 and agent 2 concentrations should be sampled, either with a checkerboard or multiple ray design. However, if a well behaved three-dimensional concentration-effect surface is anticipated, and the specific combination being studied is only one of many candidates being screened, then a single ray may be sufficient. Composite designs consisting of a checkerboard and some rays might also be used. Of course, if the intended data analysis approach is firmly tied to a particular design, then that design will have to be used.

After completing the analysis of the first mixture experiment in exploratory mode, the researcher may want to switch to confirmatory mode. The repeat of the combination experiment may use the same design as in the exploratory experiment, but probably the knowledge gained from the first run will help to refine the design for the second run. If a complex three-dimensional concentration-effect surface was found in the exploratory experiment, then agent concentrations in the interesting regions of the surface should be accented in the confirmatory experiment. Increasing the numbers of replicates probably also will be necessary. If a simple three-dimensional concentration-effect surface was found in the exploratory experiment, ie, one with pure Loewe synergism or Loewe antagonism,
TABLE 2

Central composite design (CEN)

\begin{tabular}{ccccccc}
\hline Drug 2 & \multicolumn{6}{c}{ Drug 1 concentration } \\
concentration & $\mathbf{0}$ & $\mathbf{2}$ & $\mathbf{5}$ & $\mathbf{1 0}$ & $\mathbf{2 0}$ & $\mathbf{5 0}$ \\
\hline 0 & 2 & & & 2 & 2 & 2 \\
0.2 & & & & 2 & & \\
0.5 & & & 2 & & 2 & \\
1 & 2 & 2 & & 2 & & 2 \\
2 & 2 & & 2 & & 2 & \\
5 & 2 & & & 2 & & \\
\hline
\end{tabular}

$\overline{T a b l e ~ e n t r i e s ~ a r e ~ t h e ~ n u m b e r ~ o f ~ r e p l i c a t e s ~ a t ~ e a c h ~ d e s i g n ~ p o i n t, ~ i e, ~ e a c h ~}$ combination of drug 1 concentration and drug 2 concentration

TABLE 3

Fixed ratio design with single ratio in duplicate (FR2X)

\begin{tabular}{ccccccc}
\hline Drug 2 & \multicolumn{7}{c}{ Drug 1 concentration } \\
concentration & 0 & 2 & 5 & 10 & 20 & 50 \\
\hline 0 & 2 & 2 & 2 & 2 & 2 & 2 \\
0.2 & 2 & 2 & & & & \\
0.5 & 2 & & 2 & & & \\
1 & 2 & & & 2 & & \\
2 & 2 & & & & 2 & \\
5 & 2 & & & & & 2 \\
\hline
\end{tabular}

Table entries are the number of replicates at each design point, le, each combination of drug 1 concentration and drug 2 concentration

TABLE 4

Fixed ratio design with four different ratios (FR4X)

\begin{tabular}{ccccccc}
\hline Drug 2 & \multicolumn{7}{c}{ Drug 1 concentration } \\
concentration & 0 & 2 & 5 & 10 & 20 & 50 \\
\hline 0 & 2 & 1 & 1 & 1 & 1 & 1 \\
0.1 & & 1 & & & & \\
0.2 & 1 & 1 & & & & \\
0.25 & & & 1 & & & \\
0.4 & & 1 & & & & \\
0.5 & 1 & & 1 & 1 & & \\
1 & 1 & 1 & 1 & 1 & 1 & \\
2 & 1 & & & 1 & 1 & \\
2.5 & & & 1 & & & 1 \\
4 & & & & & 1 & 1 \\
5 & 1 & & & 1 & & 1 \\
10 & & & & & 1 & 1 \\
25 & & & & & & 1 \\
\hline
\end{tabular}

Table entries are the number of replicates at each design point, ie, each combination of drug 1 concentration and drug 2 concentration 
TABLE 5

D-optimal design for absolute error, $\alpha=1$ (OPTA)

\begin{tabular}{ccccc}
\hline Drug 2 & \multicolumn{4}{c}{ Drug 1 concentration } \\
concentration & 0 & 3.52 & 6.32 & 28.4 \\
\hline 0 & 7 & 5 & & 5 \\
0.59 & 5 & & 5 & \\
0.791 & & & & \\
1.69 & 5 & & \\
\hline
\end{tabular}

Table entries are the number of replicates at each design point, ie, each combination of drug 1 concentration and drug 2 concentration

TABLE 6

D-optimal design for absolute error, $\alpha=5$ (OPTB)

\begin{tabular}{ccccc}
\hline Drug 2 & \multicolumn{4}{c}{ Drug 1 concentration } \\
concentration & 0 & 3.13 & 3.52 & 28.4 \\
\hline 0 & 7 & & 5 & 5 \\
0.445 & & 5 & & \\
0.59 & 5 & & & \\
1.69 & 5 & & \\
\hline
\end{tabular}

Table entries are the number of replicates at each design point, ie, each combination of drug 1 concentration and drug 2 concentration

then a design that facilitates the estimation of parameters with the smallest variance may be appropriate. A D-optimal design (eg, 4) may be indicated with many replicates.

Interestingly, the number of design points in a D-optimal design (Tables 5-10) is equal to the number of estimable parameters. For example, if one assumes that Equation 1, which contains six parameters, will adequately describe the three-dimensional combined action concentration-effect curve, then a D-optimal design will include only six design points, with or without replicates. The D-optimal designs may, at first, seem to be very strange and potentially noninformative. This type of frugal experimental design may have great potential for animal and human experiments, in which the experimental units are very dear. However, because D-optimal designs are very counterintuitive, a Monte Carlo computer simulation study was designed to compare several rival experimental designs, including D-optimal designs, for combined action studies.

\section{METHODS}

Monte Carlo simulations: For the Monte Carlo simulation, Equation 1 was assumed to represent the combined action of two agents, with realistic parameter values: Econ $=100 ; I C_{50,1}=10 ; m_{1}=-1 ; I C_{50,2}=1 ; m_{2}=-2$; and $\alpha=1$ (slight Loewe synergism) or $\alpha=5$ (large Loewe synergism). Ideal continuous data were generated by inserting values of $D_{1}$ and $D_{2}$ for a specific design into Equation 1 and calculating $E$ for each data point with a bisection root finder (15). The errors that were added to the ideal data to yield error-containing data were of four types: small absolute (SA); small relative with a $1 \%$
TABLE 7

D-optimal design for relative error, $\alpha=1$ (OPTC)

\begin{tabular}{ccccc}
\hline Drug 2 & \multicolumn{4}{c}{ Drug 1 concentration } \\
concentration & 0 & 118 & 95 & 1000 \\
\hline 0 & 7 & & 5 & 5 \\
3.08 & 5 & & & \\
11.8 & & 5 & & \\
1000 & 5 & & \\
\hline
\end{tabular}

Table entries are the number of replicates at each design point, ie, each combination of drug 1 concentration and drug 2 concentration

TABLE 8

D-optimal design for relative error, $\alpha=5$ (OPTD)

\begin{tabular}{ccccc}
\hline Drug 2 & \multicolumn{4}{c}{ Drug 1 concentration } \\
concentration & 0 & 28.1 & 95 & 1000 \\
\hline 0 & 7 & & 5 & 5 \\
2.85 & & 5 & & \\
3.08 & 5 & & & \\
1000 & 5 & & & \\
\hline
\end{tabular}

Table entries are the number of replicates at each design point, ie, each combination of drug 1 concentration and drug 2 concentration

coefficient of variation (CV) (SR); large relative with a $10 \%$ $\mathrm{CV}$ (LR); and large relative with a $10 \% \mathrm{CV}$ plus a noise constant equal to $1 \%$ of Econ (LC). Relative (or proportional) error is commonly found in biological systems, and a noise threshold often fixes a lower limit for acceptable measurements. Of the four error types, the LC error type is the most realistic. To generate the errors, first standard normal random numbers $(r)$ were generated via the Box-Müller Polar method (16) with the uniform $(0,1)$ random deviates coming from the IMSL subroutine, GGUBS (17). Small absolute errors were equal to $r$, small relative errors were equal to $(r / 100) \eta$, large relative errors were equal to $(r / 10) \eta$, and large relative errors plus a noise constant were equal to $(r / 10) \eta+r \beta$, where $\eta$ was the true error-free ideal dependent variable and $\beta$ was the noise constant, set to be $1 \%$ of Econ (since Econ=100, $\beta=1$ ).

Five hundred Monte Carlo datasets were generated for each type of error for each design. Equation 1 was fit to each dataset with iteratively reweighted nonlinear regression via the Nash variant (18) of the Marquardt algorithm (19). Initial parameter estimates for Econ were generated by taking an average of effects at the $(0,0)$ design point. Initial parameter estimates for $I_{550,1}$, $m_{1}$, and for $I_{50,2}, m_{2}$, were generated by fitting single agent data for drug 1 and drug 2, respectively, with a linearized transformation of Equation 2 via weighted linear regression. Initial parameter estimates for $\alpha$ were generated by rearranging Equation 1 to isolate $\alpha$ on the left side of the equals sign, then for each combination point, plugging in raw data and the other five initial parameter values to solve for $\alpha$, and finally, calculating an average $\alpha$ for the set of combination points. The 
TABLE 9

D-optimal design for relative error with intercept, $\alpha=1$ (OPTE)

\begin{tabular}{ccccc}
\hline Drug 2 & \multicolumn{4}{c}{ Drug 1 concentration } \\
concentration & 0 & 11.4 & 12.6 & 107 \\
\hline 0 & 7 & 5 & & 5 \\
1.07 & 5 & & & \\
1.40 & & & 5 & \\
3.28 & 5 & & & \\
\hline
\end{tabular}

Table entries are the number of replicates at each design point, ie, each combination of drug 1 concentration and drug 2 concentration
TABLE 10

D-optimal design for relative error with intercept, $\alpha=5$ (OPTF)

\begin{tabular}{llccc}
\hline $\begin{array}{l}\text { Drug } 2 \\
\text { concentration }\end{array}$ & 0 & \multicolumn{3}{c}{ Drug 1 concentration } \\
\hline 0 & 7 & 6.41 & 11.4 & 107 \\
0.743 & & 5 & 5 & 5 \\
1.07 & 5 & & & \\
3.28 & 5 & & & \\
\hline
\end{tabular}

Table entries are the number of replicates at each design point, ie, each combination of drug 1 concentration and drug 2 concentration

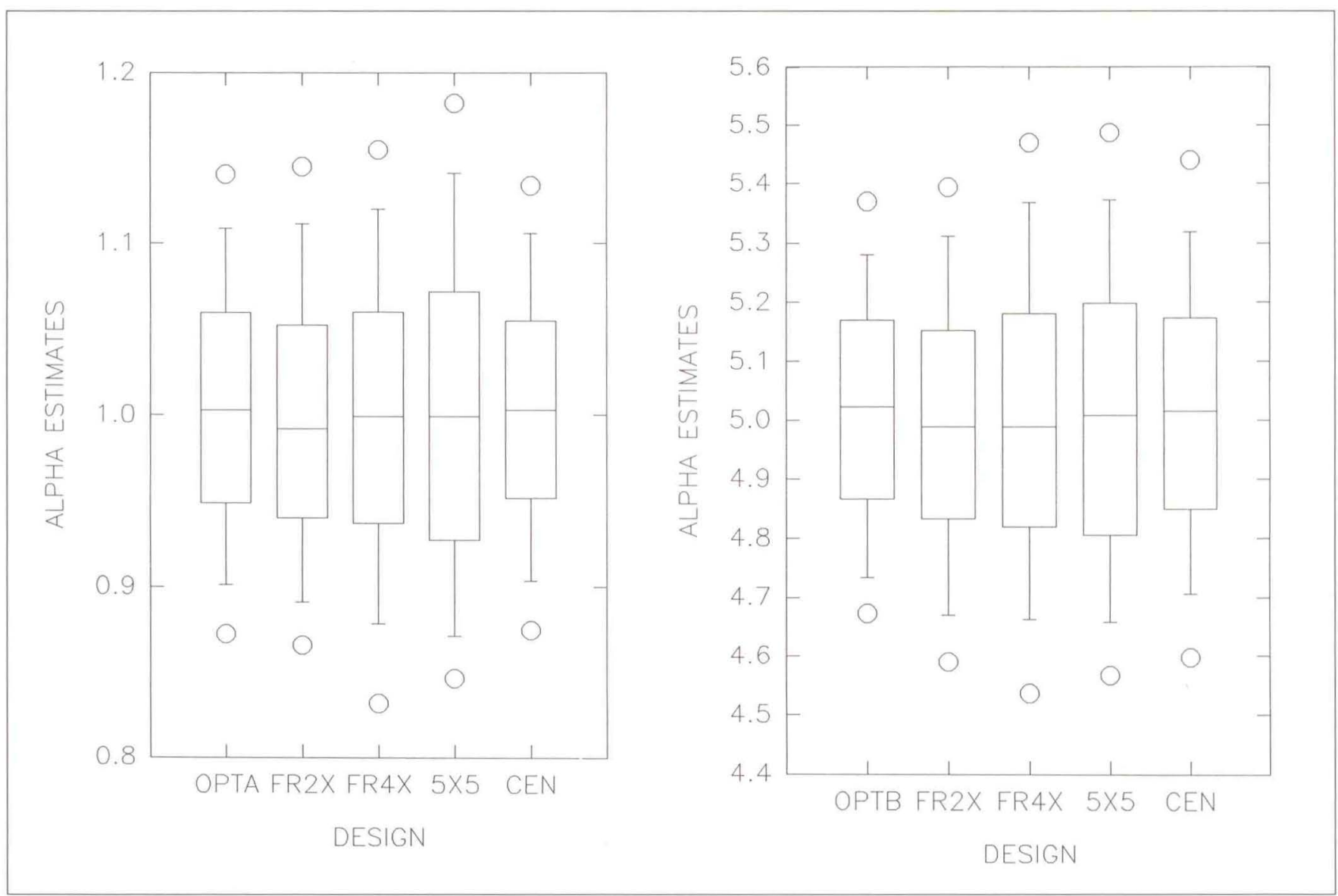

Figure 1) Box plots for the sets of $500 \alpha$ estimates for Monte Carlo data sets containing small absolute error (SA), for each of five rival designs for each of two values for the true interaction parameter $(\alpha=1, \mathbf{l e f t} ; \alpha=5$, right). The box plot for each design includes $a$ central bar for the median, upper and lower edges of a box for the 25th and 75th percentiles of the data, upper and lower lines extending to short capped bars for the 10th and 90th percentiles, and upper and lower circles which indicate the 5th and 95th percentiles

weights used for each data point were appropriate for each type of error, and were equal to 1 for $\mathrm{SA}, 1 / \hat{E}^{2}$ for $\mathrm{SR}$ and $\mathrm{LR}$, and $1 /\left[100 \beta^{2}+\hat{E}^{2}\right]$ for $\mathrm{LC}$, where $\hat{E}$ is the predicted value of $E$ for each data point at each iteration. The 100 in the denominator for the weighting factor for the LC error type is necessary to properly scale the noise constant relative to $\hat{E}$. The final parameter estimates, six for each fit of 500 datasets, were placed in files for further analyses. The generation of Monte Carlo datasets and their fitting with Equation 1 via nonlinear regression was performed with computer software written by this group in the C programming language, and run on MSDOS-compatible 80486- and 80386-based microcomputers.

Rival experimental designs: The 10 experimental designs that were compared are shown in Tables 1 to 10. All rival designs contain 32 data points. Table 1 shows a factorial or checkerboard design, with logarithmically spaced concentrations. Table 2 shows a central composite design. Table 3 shows a single fixed ratio or single ray design, in which the single fixed ratio is $10: 1$ for $D_{1}: D_{2}$ (the ratio of their $I C_{50}$ values). Table 4 shows a 

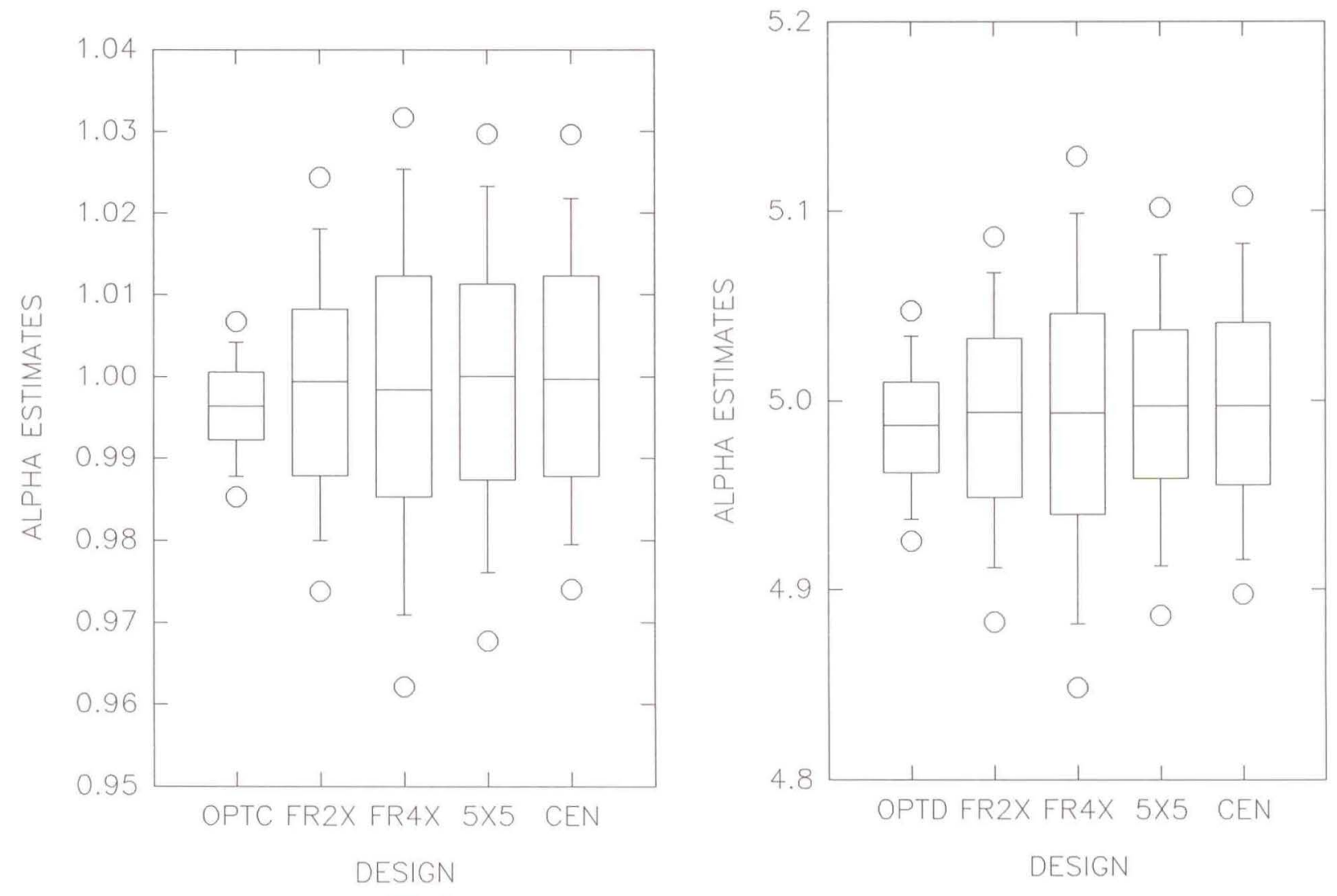

Figure 2) Box plots for the sets of $500 \alpha$ estimates for Monte Carlo data sets containing small relative error (SR), for each of five rival designs for each of two values for the true interaction parameter $(\alpha=1$, left; $\alpha=5$, right). The box plot for each design includes $a$ central bar for the median, upper and lower edges of a box for the 25th and 75th percentiles of the data, upper and lower lines extending to short capped bars for the 10th and 90th percentiles, and upper and lower circles which indicate the 5th and 95th percentiles

fixed ratio design with four different ratios (20:1, 10:1, 5:1 and 2:1). Tables 5 to 10 show D-optimal designs and are directly dependent upon the predicted values of the six parameters of Equation 1 and on the four different error structures. For each D-optimal design, the first five optimal concentration pairs $\left(D_{1}, D_{2}\right)$ (those not associated with the estimation of the interaction parameter, $\alpha$ ) were generated with formulas and concepts published by Bezeau and Endrenyi (20) for the precise estimation of the three single agent parameters for Equation 2. A discussion of the generation of D-optimal designs for the sixth optimal concentration pair (the one associated with the estimation of $\alpha$ ) for combined action studies with numerical function minimization methods has been published by the present group (4). However, the $\mathrm{D}$-optimal designs in Tables 5 to 10 were generated with faster algorithms, which relied heavily on partial analytical solutions of the determinants of the variancecovariance matrix of parameters, for which the details will be published elsewhere. The D-optimal formulas were coded in the Mathematica mathematical programming language (21). All concentrations calculated to be greater than 1000 were capped at 1000 to keep the D-optimal designs realistic.

\section{RESULTS}

Figures 1 to 4 show box plots of the distributions of the sets of estimated $\alpha$ parameters. Each figure is for a different error type. Figure 1 is for SA, Figure 2 is for SR, Figure 3 is for LR and Figure 4 is for LC. These figures were made with the Sigma Plot graphics package (22). Table 11 lists the variances for the set of $500 \alpha$ estimates for each design for each type of error (SA, SR, LR or LC) with each true $\alpha$ ( 1 or 5). For each type of error and each value of the true $\alpha$, the designs are listed in the order of increasing variance for the sets of $500 \alpha$ estimates. Each set of 500 estimates was also divided into 20 consecutive subsets of 25 values, and the variance for each subset was calculated. Then these sets of 20 variance values were used to make pairwise comparisons of variance among all rival models in a group using the Wilcoxon rank sum test via the SAS statistical package (23).

Since each group of designs involved 10 comparisons, the type I error rate of 0.01 was chosen for making decisions of statistical significance for pairwise design differences, for an overall Bonferroni conservative type I error rate of 0.1 per group. For each group, the upper designs with the smallest variance form a subgroup, for 

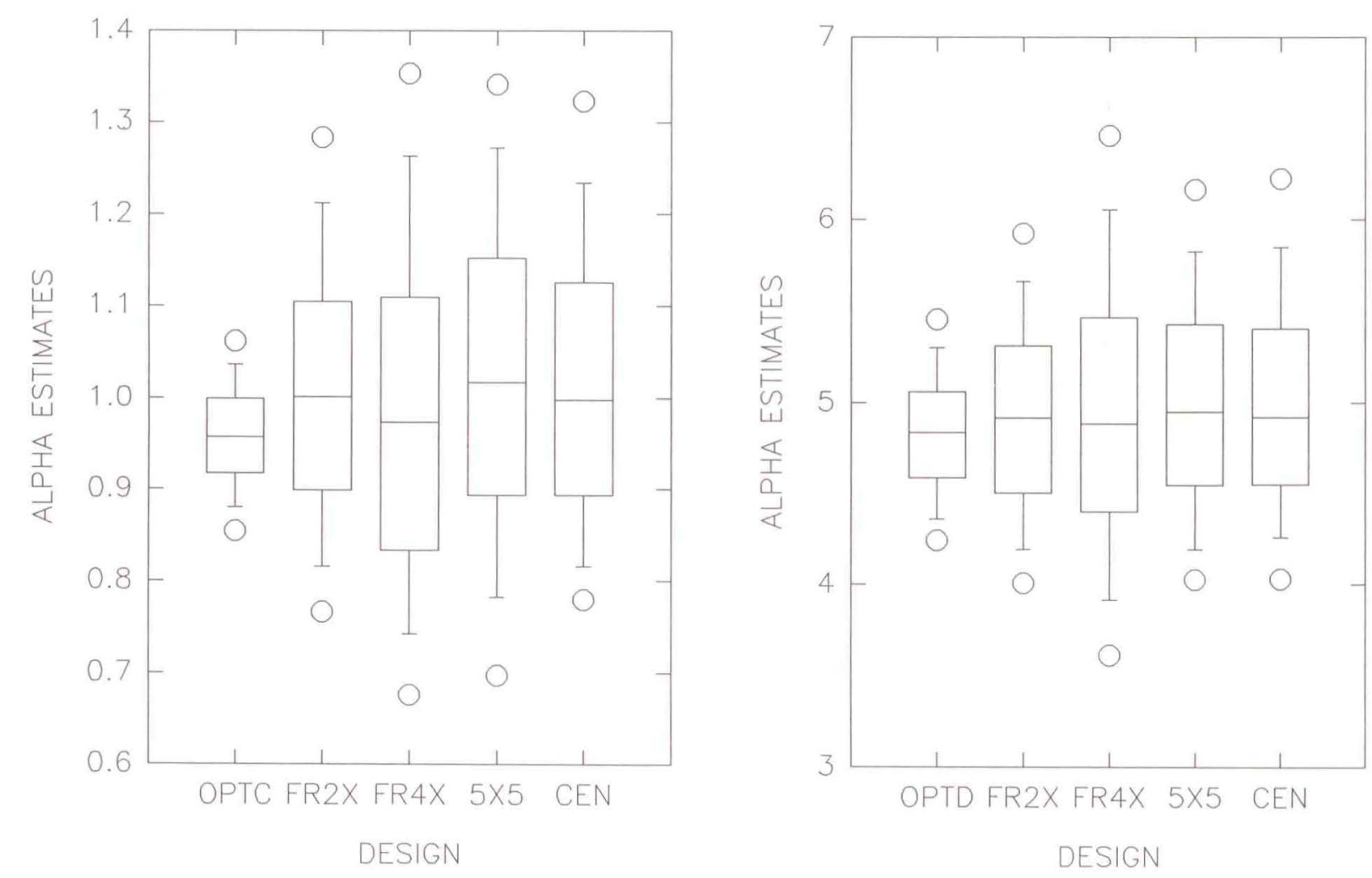

Figure 3) Box plots for the sets of $500 \alpha$ estimates for Monte Carlo data sets containing large relative error (LR), for each of five rival designs for each of two values for the true interaction parameter ( $\alpha=1$, left; $\alpha=5$, right). The box plot for each design includes a central bar for the median, upper and lower edges of a box for the 25th and 75th percentiles of the data, upper and lower lines extending to short capped bars for the 10th and 90th percentiles, and upper and lower circles which indicate the 5th and 95th percentiles

which the top design was not found to be significantly different from the other designs in the subgroup. For each subgroup, the lower designs with the largest variances form a subgroup, for which the bottom design was not found to be significantly different from the other designs in the subgroup. Significant biases were determined by comparing the mean of each set of 500 $\alpha$ estimates with the true $\alpha$ via the Student's $t$ test with a type I error rate of 0.01 with SAS.

A few general conclusions can be derived from Figures 1 to 4 and Table 11 . First, for every group, the D-optimal designs were always among the design subgroup with the smallest variance. The superiority of the D-optimal designs over all of the other designs is clearly seen for the SR, LR and LC error types, but is less clear for SA, especially for the case in which the true $\alpha=1$. Second, the FR4X design (Table 4) was always in the subgroup with the largest variance. Third, there was a tendency for the FR2X design (Table 3 ) to be a relatively precise design and for 5x5 (Table 1) to be a relatively imprecise design. Fourth, the CEN design (Table 2) tended to be very precise for the SA error type, but had medium precision for the SR, LR and LC error types. Even though OPTC (Table 7), OPTD (Table 8), OPTE (Table 9) and OPTF (Table 10) had the smallest variances for the SR, LR, and LC error types, respectively, these designs resulted in a significant bias.

\section{DISCUSSION}

Among the five rival experimental designs, the seemingly counterintuitive D-optimal designs appear to result in the smallest variance for the interaction parameter, $\alpha$. From this result, one can infer that the D-optimal designs may be superior for assessing quantitatively the nature and intensity of combined action of two agents. The convenient single fixed ratio in duplicate design (FR2X, Table 3) was also very good, and ranked second.

Formal statistical experimental design often includes an interesting paradox: to design an experiment well, one has to know the final answer well. However, if the final answer is well known, ie, both the correct model and the true model parameters, then one would not have to conduct the experiment. This paradox is solved with sequential experimentation; each experiment in a sequence provides better information for the 

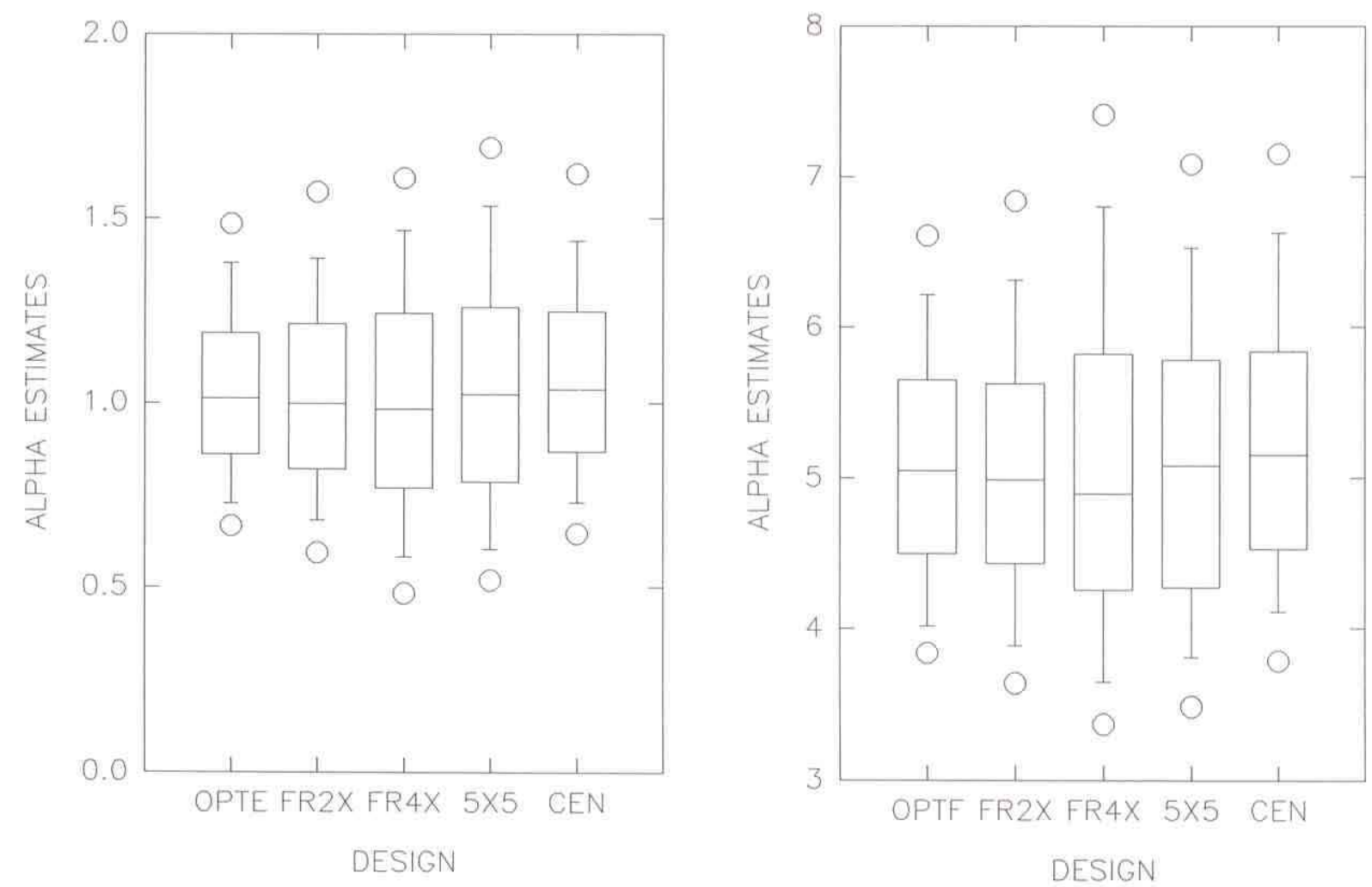

Figure 4) Box plots for the sets of $500 \alpha$ estimates for Monte Carlo data sets containing large relative error with a noise constant (LC). for each of five rival designs for each of two values for the true interaction parameter ( $\alpha=1$, left: $\alpha=5$, right). The box plot for each design includes a central bar for the median, upper and lower edges of a box for the 25th and 75th percentiles of the data, upper and lower lines extending to short capped bars for the 10th and 90th percentiles, and upper and lower circles which indicate the 5 th and 95th percentiles

\section{TABLE 11}

Variances for distributions of $\alpha$ parameter estimates

\begin{tabular}{|c|c|c|c|c|c|c|c|c|}
\hline \multirow[b]{3}{*}{ True $\alpha$} & \multicolumn{8}{|c|}{ Error type } \\
\hline & \multicolumn{2}{|c|}{ Small absolute (SA) } & \multicolumn{2}{|c|}{ Small relative (SR) } & \multicolumn{2}{|c|}{ Large relative (LR) } & \multicolumn{2}{|c|}{$\begin{array}{c}\text { Large relative }+ \\
\text { noise constant }(\mathrm{LC})\end{array}$} \\
\hline & Design & Variance & Design & Variance & Design & Variance & Design & Variance \\
\hline \multirow[t]{5}{*}{1} & $\mathrm{CEN}^{\dagger}$ & 0.00619 & OPTC ${ }^{+}$ & 0.0000409 & OPTC*t & 0.00380 & OPTE ${ }^{+}$ & 0.0629 \\
\hline & OPTA $^{\dagger}$ & 0.00659 & FR2X & 0.000236 & FR2X & 0.0246 & FR2X & 0.0913 \\
\hline & FR2X ${ }^{\dagger}$ & 0.00763 & CEN & 0.000301 & CEN & 0.0307 & CEN* & 0.0962 \\
\hline & FRAX $X^{\neq}$ & 0.00900 & $5 \times 5^{\neq}$ & 0.000356 & $5 \times 5^{+}$ & 0.0384 & FRA $4 X^{*}$ & 0.121 \\
\hline & $5 \times 5^{+}$ & 0.0110 & FRAX $X^{\neq}$ & 0.000427 & FR4X ${ }^{\ddagger}$ & 0.0411 & $5 \times 5^{\dagger}$ & 0.129 \\
\hline \multirow[t]{5}{*}{5} & OPTB $^{\dagger}$ & 0.0463 & OPTD* ${ }^{*}$ & 0.00134 & OPTD ${ }^{+}$ & 0.129 & OPTF*t & 0.727 \\
\hline & $\mathrm{CEN}^{\dagger}$ & 0.0605 & FR2 $2 X^{*}$ & 0.00395 & FR2X* & 0.345 & FR2 $X^{\dagger}$ & 0.937 \\
\hline & FR2 $X^{\ddagger}$ & 0.0611 & $5 \times 5$ & 0.00409 & $5 \times 5$ & 0.405 & CEN* & 1.15 \\
\hline & FRAX $X^{\dagger}$ & 0.0764 & CEN & 0.00430 & CEN & 0.449 & $5 \times 5^{\dagger}$ & 1.26 \\
\hline & $5 \times 5^{4}$ & 0.0790 & FR4 $X^{\neq}$ & 0.00723 & FRAX ${ }^{\ddagger}$ & 0.752 & $\mathrm{FR} \mathrm{X}^{\ddagger}$ & 1.44 \\
\hline
\end{tabular}

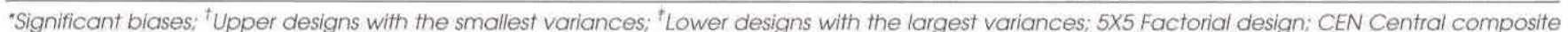

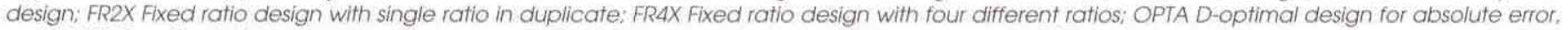

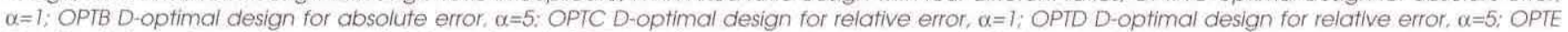
D-optimal design for relative error with intercept, $\alpha=1$; OPTF D-optimal design for relative error with intercept, $\alpha=5$ 
planning of the subsequent experiment. Based upon the results of the present study, for confirmatory twoagent combined action Loewe synergism studies for pairs of agents with simple three-dimensional concentration-effect surfaces (appropriately modelled with Equation 1), one should: first, understand the concentration-effect curves of each individual agent well, ie,

ACKNOWLEDGEMENTS: This work was supported by NIH grants CA46732 and CA16056.

\section{REFERENCES}

1. Syracuse KC, Greco WR. Comparison between the method of Chou and Talalay and a new method for the assessment of the combined effects of drugs: A Monte-Carlo simulation study. Proc Am Stat Assoc Biopharm Sect 1986:127-32.

2. Greco WR, Lawrence DL. Assessment of the degree of drug interaction where the response variable is discrete, Proc Am Stat Assoc Biopharm Sect 1988:226-31.

3. Greco WR. Importance of the structural component of generalized nonlinear models for joint drug action. Proc Am Stat Assoc Biopharm Sect 1989:183-8.

4. Greco WR, Tung Y. D-optimal experimental designs for quantifying synergy in drug combination studies. Proc Am Stat Assoc Biopharm Sect 1991:244-9.

5. Greco WR. The use of LEGO bricks to construct solid 3-dimensional dose-response surfaces. 23rd Symposium on the Interface of Computing Science and Statistics, Interface 91. Proceedings. 1991:326-31.

6. Greco WR, Park HS, Rustum YM. An application of a new approach for the quantitation of drug synergism to the combination of cis-diamminedichloroplatinum and 1- $\beta$-D-arabinofuranosylcytosine. Cancer Res 1990;50:5318-27.

7. Gaumont Y, Kisliuk, RL, Parsons JC, et al. Quantitation of folic acid enhancement of antifolate synergism. Cancer Res 1992;52:2228-35.

8. Greco WR, Dembinski WE. Fundamental concepts in the assessment of the joint interaction of biological response modifiers with other agents. Can J Infect Dis 1992;3(Suppl B):60B-8B.

9. Greco WR, Rustum YM. Reply to letters by Berenbaum and Sühnel concerning Greco et al. (Cancer Res 1990;50:5318-27) Cancer Res 1992;52:4561-5. (Lett) obtain precise estimates of $I_{50.1}, m_{1}, I C_{50.2}$, and $m_{2}$; second, use a single ray fixed ratio design for combined action to get a fair estimate of $\alpha$; and, finally, use a D-optimal design for a precise estimate of $\alpha$. Alternatively, one might want to use a standard fixed ratio or factorial design routinely, but then also add the D-optimal design points to the final experimental design.

10. Carter WH Jr, Gennings C, Staniswalis JG, et al. A statistical approach to the construction and analysis of isobolograms. J Am Coll Toxicol 1988;7:963-73.

11. Berenbaum MC. The expected effect of a combination of agents: The general solution. J Theor Biol 1985;114:413-31

12. Greco WR, Unkelbach HD, Pöch G, Sühnel J, Kundi M, Bödeker W. Consensus on concepts and terminology for interaction assessment: The Saaresilkä Agreement. Arch Complex Environ Stud 1992;4:65-9.

13. Loewe S, Muischnek H. Effect of combinations; mathematical basis of problem. Arch Exp Path Pharmak 1926; 114:313-26.

14. Hill AV. The possible effects of the aggregation of the molecules of haemoglobin on its dissociation curves. J Physiol 1910;40:iv-vii.

15. Thisted RA. Elements of Statistical Computing. New York: Chapman and Hall, 1988:170.

16. Box GEP, Müller ME. A note on the generation of normal deviates. Ann Math Stat 1958;29:610-1.

17. Lewis PAW, Goodman AS, Miller JM. Pseudo-random number generator for the system/360. IBM Systems $J$ 1969;8:136-46.

18. Marquardt DW. An algorithm for least squares estimation of nonlinear parameters. J Soc Ind Appl Math 1963;11:431-41.

19. Nash JC. Compact Numerical Methods for Computers: Linear Algebra and Function Minimisation. New York: John Wiley \& Sons, 1979:175-7.

20. Bezeau M, Endrenyi L. Design of experiments for the precise estimation of dose-response parameters: The Hill equation. J Theor Biol 1986; 123:415-30.

21. Wolfram S. Mathematica: A System for Doing Mathematics by Computer. Redwood City: Addison-Wesley Publishing Co, 1988.

22. Kuo J, McDonald S, Fox E. Sigma Plot Users Manual. San Rafael: Jandel Scientific, 1992.

23. SAS Institute Inc. SAS/STAT Guide for Personal Computers, Version 6. Cary: SAS Institute, 1987. 


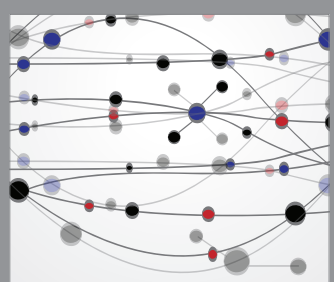

The Scientific World Journal
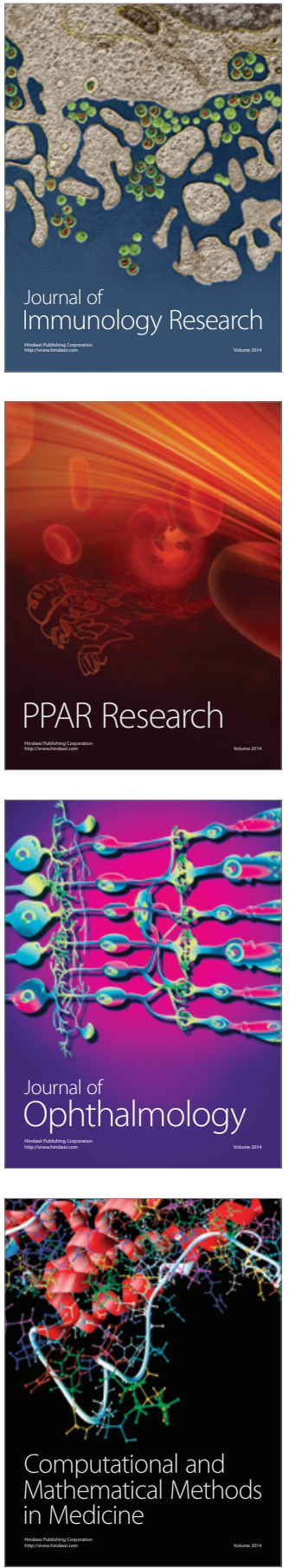

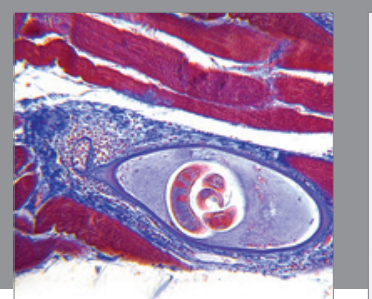

Gastroenterology Research and Practice

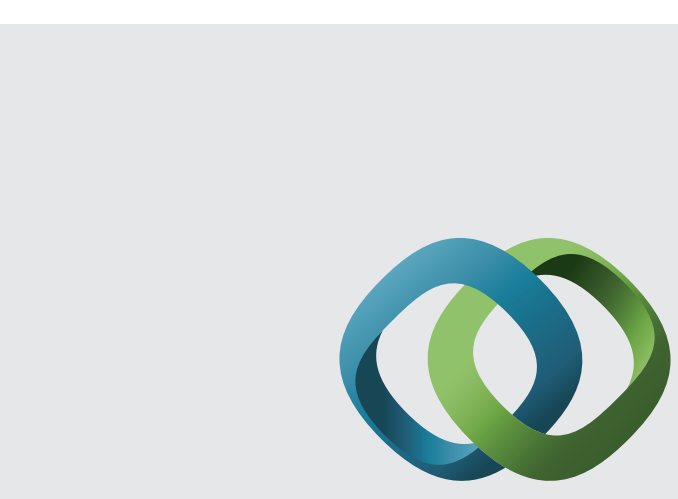

\section{Hindawi}

Submit your manuscripts at

http://www.hindawi.com
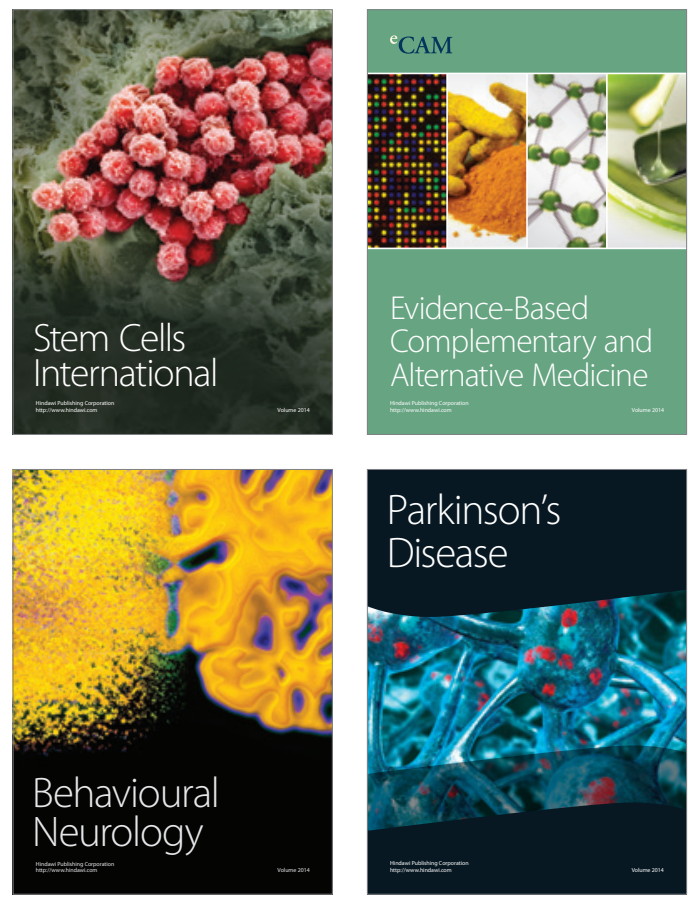
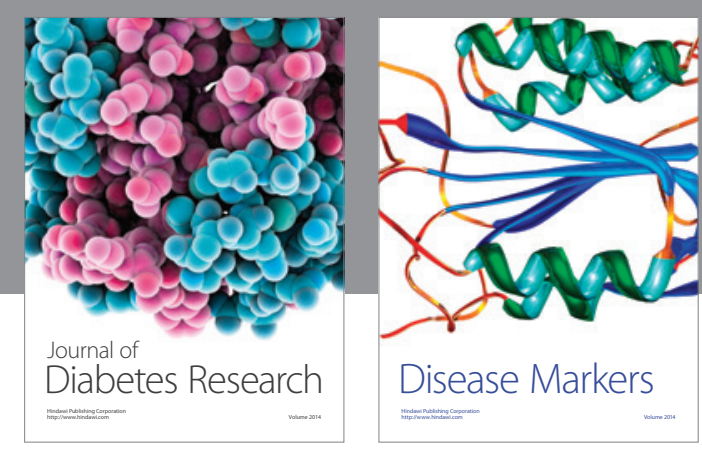

Disease Markers
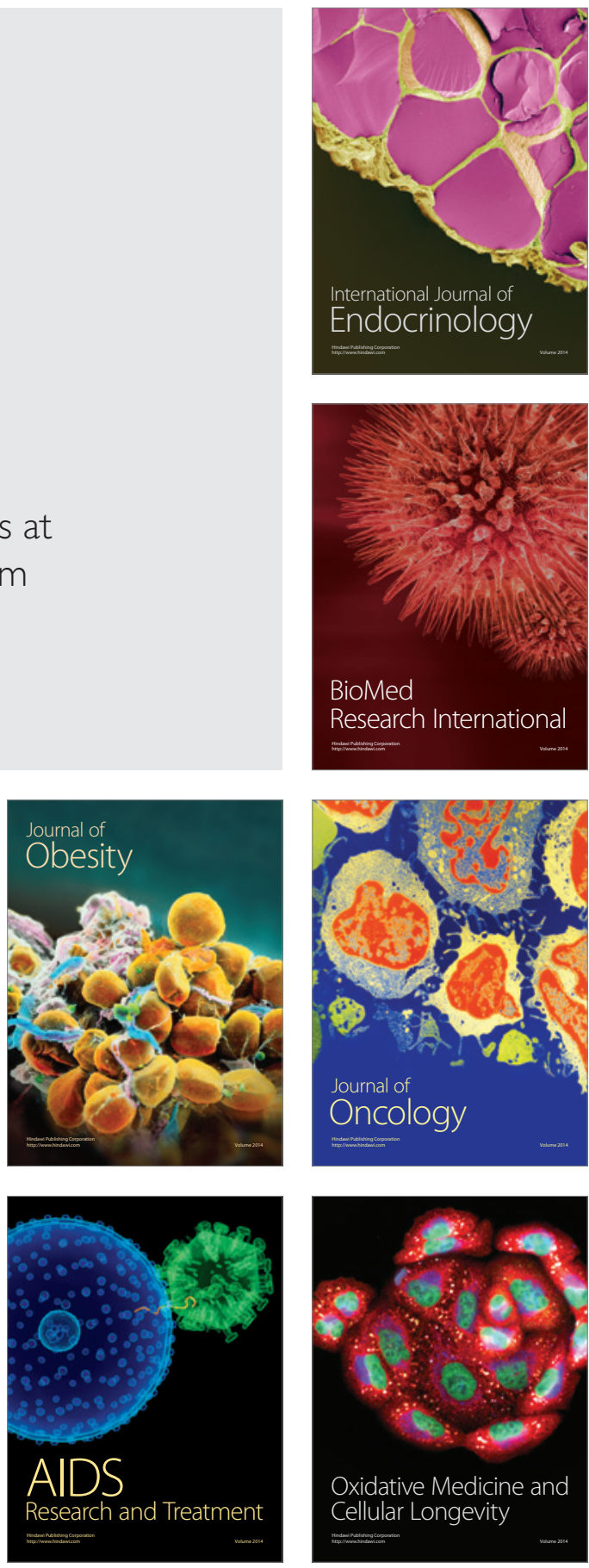